\title{
Microcontact printing of metalloproteins
}

\author{
Adriana Biasco *, Dario Pisignano, Blandine Krebs, Roberto Cingolani, Ross Rinaldi \\ NNL, National Nanotechnology Laboratory of Istituto Nazionale di Fisica della Materia (INFM), c/o Dipartimento di Ingegneria \\ dell'Innovazione, Università di Lecce, via Arnesano, I-73100 Lecce, Italy
}

\begin{abstract}
The morphological investigation by scanning probes techniques, and especially Atomic Force Microscopy (AFM) allow to measure the surface roughness of immobilized proteins with unequalled vertical spatial resolution. This is particularly important, since the biological role of proteins is closely related to their physiological folding architectures. In particular, metalloproteins, such as blue copper proteins, revealed to be good candidates for biomolecular electronics by virtue of their natural electron transfer activity, which is exploitable in molecular switches whose conduction state can be controlled by gating their redox state. The interest in these molecules is indeed motivated by the possibility of eliciting current flow through the redox level of single molecules. Printing metalloproteins can be an advantageous approach for the easy realization of bioelectronic circuits. We implemented and developed the selective deposition of the metalloprotein Azurin (Az) from Pseudomonas aeruginosa by microcontact printing, and investigated by AFM the surface morphology of the printed Az layers.
\end{abstract}

Keywords: Metalloproteins, microcontact printing, self-assembly using surface chemistry, atomic force microscopy,

\section{Introduction}

Several applications in biology require proteins immobilized on substrates. Among them, particular interest is being aroused by microarray fabrication $[1,2]$ and biomolecular electronics $[3,4]$. In both these fields, the possibility of patterning proteins within specific locations in two-dimensional structures, possibly with $\mu \mathrm{m}$-scale or sub- $\mu \mathrm{m}$-scale controlled resolution, is a desirable goal [5, 6], in order to control the array distribution in protein chips.

Proteins are biological macromolecules constructed from one or more chains of amino acids linked by peptide bonds and folded into a specific three-dimensional configuration (tertiary structure). High-throughput proteinbased technologies have to preserve this three-dimensional structure and, therefore, the activity and specificity of the employed biomacromolecules. We underline that, for instance, the fabrication of protein chip requires much more efforts, than DNA chip; in fact, while the DNA is a relatively robust molecule, consisting of only four different building blocks, proteins are typically more difficult to

\footnotetext{
* Also at ISUFI, Università di Lecce, via Arnesano, I-73100 Lecce, Italy. Corresponding author. Tel: +39-0832-298378; fax: +39-0832298238; E-mail: adriana.biasco@unile.it
}

handle and a narrower range of environmental conditions (temperature, $\mathrm{pH}$, solvent ionic force, etc.) are needed to deliver them as functional entities [7].

By virtue of their structural and functional properties, proteins are also considered good candidates as building blocks in the bottom-up fabrication of biosensors and hybrid molecular electronic devices. In particular, metalloproteins, such as blue copper proteins, revealed to be good candidates for biomolecular electronics because of their natural electron transfer activity, which is exploitable in molecular switches whose conduction state can be controlled by gating their redox state [8]. The interest in these molecules is indeed motivated by the possibility of eliciting current flow through the redox level of single molecules. Therefore, selectively patterning metalloproteins can be an advantageous approach for the easy realization of bioelectronic circuits.

For all these reasons, specific processing and patterning techniques are required, preserving proteins and preventing them from binding in a non-specific way, which would lead to protein chips with a high signal-to-noise ratio. So far, several techniques have been developed for patterning proteins on pre-modified substrates, such as photochemical methods [9], photografting, inkjet printing [10] and printing robots [11]. Nevertheless, soft lithography [12] is by far considered the most convenient approach 


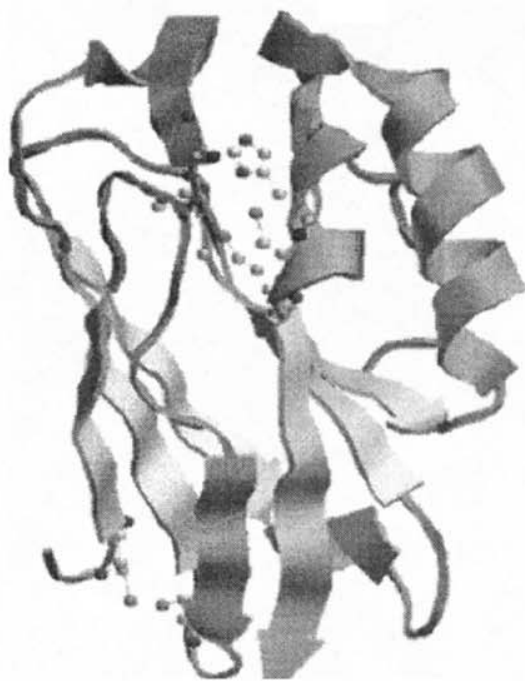

Fig. 1 Molecular structure of the Az protein.

because of its experimental simplicity, low cost, and extreme flexibility in terms of the employable substrates, solvents, and deliverable molecules. However, although much work has been done to achieve high-throughput direct patterning of biomolecules onto surfaces, the generality of the approach to produce patterns of azurin by microcontact printing $(\mu \mathrm{CP})$ is still unknown.

In this work, we implemented and developed the selective deposition of the metalloprotein Azurin (Az, molecular structure in Fig. 1) from Pseudomonas aeruginosa by microcontact printing. We assessed the successful transfer of the biomolecules by immunofluorescence experiments, and investigated the surface morphology of the printed Az layers by atomic force microscopy (AFM). Our approach is based on the modification of the substrate surface properties by introducing a surface linker (-SH functional group) that allows a site-specific binding of the protein.

\section{Experimental}

$\mathrm{Az}$ and the anti-Az serum (primary antibody) were kindly supplied by the metalloprotein groups, Gorlaeus Laboratory, Leiden, The Netherlands. It was diluted in 20 $\mathrm{mM}$ Hepes buffer, $\mathrm{pH} 4.6$, to a final concentration of $10^{-8}$ $\mathrm{M}$ and $10^{-5}$ M. 3-Mercapto Propyl Trimethoxysilane (3MPTS) was purchased from Sigma and was diluted immediately prior to use to $2 \%(\mathrm{v} / \mathrm{v})$ in absolute ethanol. Secondary antibody (1:32 rhodamine-labelled goat antirabbit IgG antibody) was purchased from Sigma-Aldrich.

All the microfabrication steps were performed in a 1000-class clean room. $\mathrm{SiO}_{2}$ wafers were cleaved into 1 by $1 \mathrm{~cm}^{2}$ pieces; they were pre-cleaned using a $3: 1$ mixture of $\mathrm{H}_{2} \mathrm{SO}$ and $\mathrm{H}_{2} \mathrm{O}_{2}$ in order to remove organic contaminants and sonicated three times for 5 minutes in water at $25^{\circ} \mathrm{C}$. The substrates were then dried with a blast of $\mathrm{N}_{2}$ gas to remove the bulk water thus preventing undesirable polymerization of the 3-MPTS. All the surfaces were used immediately after cleaning. To introduce - $\mathrm{SH}$ functional groups on the surface, silanization was carried out in a $2 \%$ (v/v) solution of 3-MPTS in ethanol at $25{ }^{\circ} \mathrm{C}$ for 15 minutes. Sonication in ethanol was then performed for 1 minute, to remove eventually non-covalently adsorbed silane compounds, and then dehydration was carried out with a $\mathrm{N}_{2}$ flux. The so treated substrates were stored under vacuum until the contact with protein.

The performed $\mu \mathrm{CP}$ lithography process is schematized in Fig. 2. The master patterns, made by parallel stripes of periodicity between 20 and $30 \mu \mathrm{m}$ were fabricated by photolithography on $\mathrm{Si} / \mathrm{SiO}_{2}\left(\mathrm{SiO}_{2}\right.$ thickness $\left.\cong 100 \mathrm{~nm}\right)$ substrates. After the deposition of standard photoresist (Clariant AZ5214E:AZ EBR Solvent 1:1), UV-exposure (about 8 minutes at $45 \mathrm{~W}$ ) and developing, we etched the
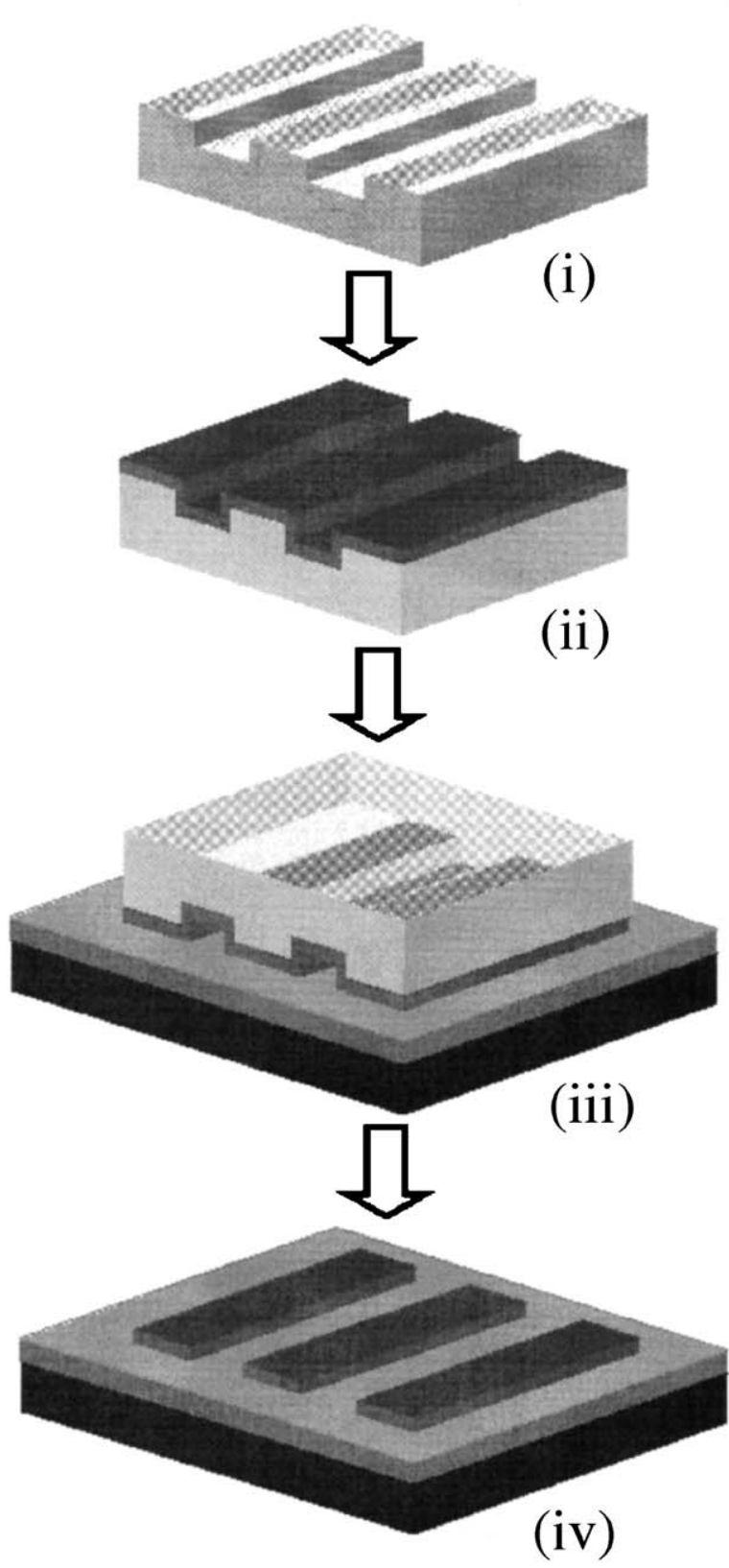

Fig. 2 Scheme of the $\mu$ CP. The stamp, obtained by casting the elastomer over master (i), is inked by the protein solution (ii). It is then placed onto the functionalised substrate (iii), and then peeled off, thus forming a protein layer on the substrate surface (iv). 
thermal dioxide by using a $\mathrm{NH}_{4} \mathrm{~F} / \mathrm{HF} / \mathrm{H}_{2} \mathrm{O}$ solution. The so obtained $\mathrm{SiO}_{2}$ mask was then employed to etch Silicon by $5.9 \mathrm{M}$ solutions of $\mathrm{KOH}$ at $80^{\circ} \mathrm{C}$. Elastomeric replicas of hard masters are realized by in situ polymerization of poly(dimethylsiloxane) (PDMS) (Sylgard 184, A:B 1:9, Dow Corning) at $140^{\circ} \mathrm{C}$ for 15 minutes. The patterned side of the replica was exposed to a $10^{-8}-10^{-5} \mathrm{M}$ solution of the protein and gently dried with a $\mathrm{N}_{2}$ stream. Immediately after the solvent evaporation, the resulting protein-coated elastomeric element was placed onto the modified substrates under its own weight. The replicas were peeledoff and the resulting patterned substrates were vigorously rinsed several times in order to remove the unattached protein molecules. The samples for immunofluorescence were then incubated with a anti-Az serum for 45 minutes, and washed three times with phosphate buffer saline. Then they were incubated for 45 minutes with the secondary antibody and washed again. They were finally dried and immediately observed under the fluorescence microscope.

The protein-patterned images were observed using an inverted fluorescence microscope fitted with a monochrome camera AxioCam MR (Zeiss). AFM experiments were conducted with a BioScope microscope (Digital Instruments) equipped with a $90 \mu \mathrm{m}$ scanner and operating in air tapping mode. $\mathrm{Si}_{3} \mathrm{~N}_{4}$ tips with a $10 \mathrm{~nm}$ nominal curvature radius were used with a scanning rate of $1 \mathrm{~Hz}$.

\section{Results and discussion}

The immunofluorescence analysis shows that the irreversible patterned immobilization of metalloprotein $\mathrm{Az}$ was pursued on silanized $\mathrm{SiO}_{2}$. Indeed, after patterning the proteins and after polyclonal antibody incubation, sets of ordered, $\sim 9 \mu \mathrm{m}$ wide parallel tracks could be observed (Fig. 3 ), corresponding to the width of the recessed grooves of the PDMS mold.

The non-specific adsorption of a soluble proteins onto the target surface could be a relevant problem for analytical applications, which are based on the specific interactions between proteins in solution and surface-bound molecules. For this reason, it is particularly important to protect the surface from non-specific binding on the non-patterned area. The high signal-to-noise ratio (i.e. intensity of light collected from the pattern / background light intensity) observed by immunofluorescence indicating that our $\mathrm{Az}$ almost does not bind to regions of the substrate between the tracks. This indicates that a highly specific binding of the

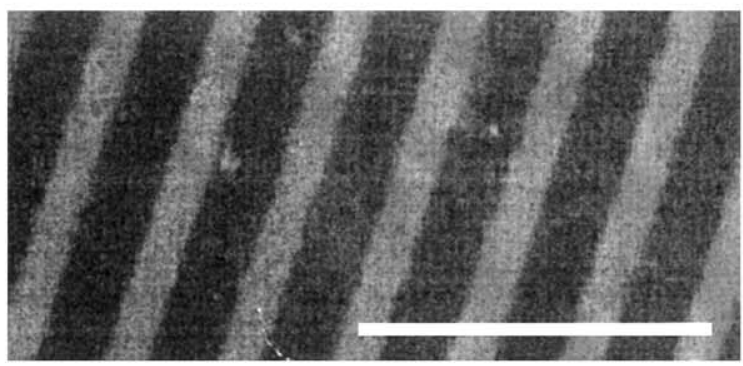

Fig. 3 Immunofluorescence micrograph of the obtained Az pattern. The white bar indicates a $100 \mu \mathrm{m}$ length. metalloproteins is achieved with $\mu \mathrm{CP}$, by virtue of both the excellent conformal contact provided by the PDMS replica and the negligible lateral diffusion of the macromolecules on the target surface (which is also confirmed by the absence of proteins in the inter-stripe regions founded by the subsequent AFM investigation). The very small amount of fluorescence collected from the un-printed regions of the sample can be likely attributed to the non-specific (passive) adsorption of the primary antibody onto the bare substrate.

Besides the specificity, the $\mathrm{SiO}_{2}$ surface was opportunely modified to immobilize the target protein in a desired molecular orientation. Indeed, the controlled tailoring of the substrate surface properties was pursued by using a surface organic modifier (silane) that can chemically bind the protein and that forms a uniform monolayer with root mean square (rms) roughness of 0.3 $\mathrm{nm}$ [13]. The Az proteins adsorb onto the silane layer via a disulfide bond. Given to this highly binding specificity, proteins interact with the surface by a chemically driven narrow distribution of orientations.

The optimal Az concentration for collecting a bright fluorescence signal was found to be $10^{-5} \mathrm{M}$. For concentrations lower than $10^{-6} \mathrm{M}$ the adsorption process still provides an effective pattern transfer, as shown by the AFM inspection (Fig. 4), but the fluorescence emission intensity is not sufficient to be detected by our set-up. The protein concentration $\left(10^{-8} \mathrm{M}\right)$ employed for the AFM imaging was lowered, since less diluted solutions gave rise to molecular aggregates formed all over the surface (and still present after washing). This clearly indicate a significant mutual interaction among the protein molecules.

Globular proteins like $\mathrm{Az}$ are stabilized in solution by intramolecular forces that maintain their three-dimensional conformation. It is largely accepted that the contact with solid surfaces can lead to protein denaturation. Indeed, it has been demonstrated that these conformational changes range from slight shape modifications to possibly complete denaturation, occurring on timescales between a few minutes and some hours. [14, 15]. Moreover, the denaturation process is thought to be more effective onto hydrophobic surfaces than onto hydrophilic ones [16]. The

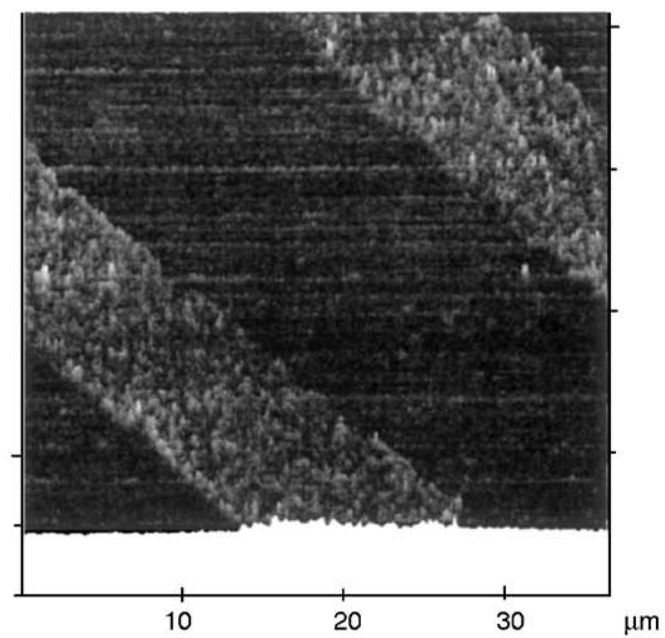

Fig. 4 Topography image of a printed $\mathrm{Az}$ layer by $10^{-8} \mathrm{M}$ solutions. 
extent to which the protein loses its own helicity upon adsorption is highly dependent on the individual nature of the protein and the surface itself. In the $\mu \mathrm{CP}$ case, a third factor playing an important role is the possible alteration of conformation induced by the surface properties of the elastomeric surfaces (hydrophobic/hydrophilic interactions). Of course, the simple inspection by immufluorescence optical microscopy does not allow to observe the single-protein conformational properties. Scanning force microscopy enabled instead the investigation of protein adsorption at a surface by imaging single biomacromolecules with precise molecular sizing. Further studies are in progress to assess the possible morphological and functional variations induced by the patterning process on the metalloproteins.

\section{Conclusion}

In conclusion, we specifically implemented the $\mu \mathrm{CP}$ technique for patterning metalloproteins with a low-cost and parallel approach. The resulting protein patterns were observed by immunofluorescence optical microscopy, demonstrating the good contrast achievable by the lithographic processes. More important, the investigation of the printed proteins onto silanised $\mathrm{SiO}_{2}$ surfaces by $\mathrm{AFM}$ scanning probes allowed us to exclude the occurrence of protein diffusion in the inter-stripes regions and to assess the optimal solution concentration to achieve printed monolayers, thus opening the way to the possible realisation of controlled patterns of metalloproteins for bioelectronic circuitry.
Acknowledgments Support for this research was provided by the Italian Minister of University and Scientific Research (FIRB-Nanodispositivi Molecolari Prot. RBNEO1YSR8), and by the EU (SAMBA Project).

\section{References}

[1] M. F. Lopez, M. G. Pluskal, J. Chromatogr. B 787 (2003) 19.

[2] A. Lueking, M. Horn, H. Eickhoff, K. Bussow, H. Lehrach, G. Walter, Anal. Biochem. 270 (1999) 103.

[3] A. S. Martin, J. R. Sambles, Phys. Rev. Lett. 1993, 70, 218. C. Joachim, J. Gimzewski, A. Aviram, Nature 408 (2000) 541.

[4] R. Rinaldi, A. Biasco, G. Maruccio, R. Cingolani, D. Alliata, L. Andolfi, P. Facci, F. De Rienzo, R. De Felice, E. Molinari, Adv. Mater. 14 (2002) 1453.

[5] G. MacBeath, S. L. Schreiber, Science 289 (2000) 1760.

[6] H. Zhu, M. Bilgin, R. Bangham, D. Hall, A. Casamayor, P. Bertone, N. Lan, R. Jansen, S. Bidlingmaier, T. Houfek, T. Mitchell, P.Miller, R A. Dean, M. Gerstein, M. Snyder, Science 293 (2001) 2101

[7] H. Eickhoff, Z. Konthur, A. Lueking, H. Lehrach, G. Walter, E. Nordhoff, L. Nyarsik, K. Bussow, Adv Biochem Eng Biotechnol. 77 (2002) 103.

[8] R. Rinaldi, R. Cingolani, Physica E 21 (2004) 45.

[9] A. S. Blawas, W.M. Reichert, Biomaterials 19 (1998) 595.

[10] ] P. Calvert, Chem. Mat. 13 (2001) 3299.

[11] G. MacBeath, S. L. Schreiber, Science 289 (2000) 1760

[12] Y. Xia, G. M. Whitesides, Angew. Chem. Int. Ed. 37 (1998) 550.

[13] A. Biasco, G. Maruccio, P. Visconti, A. Bramanti, P. Calogiuri, R. Cingolani, R. Rinaldi, Mater. Sci. Eng. C 24 (2004) 563.

[14] A. M. Moulin, S. J. O'Shea, R. A. Badley, P. Doyle, M. E. Welland, Langmuir 15 (1999) 8776.

[15] S. A. Holt, D. J. McGillivray, S. Poon, J. W. White, J. Phys. Chem. B 104 (2000) 7431

[16] J. J. Gray, Curr. Opin. Struct. Biol. 14 (2004) 110. 